\title{
Reconnection flow jets in 3D as a source of structured dipolarization fronts
}

\author{
Philip L. Pritchett
}

\begin{abstract}
Three-dimensional electromagnetic particle-in-cell simulations are used to investigate the propagation and breakup of a reconnection flow jet of initial cross-tail extent $24 d_{i}\left(\sim 1.5 R_{E} ; d_{i}\right.$ is the ion inertial length). Such a front is found to separate into two segments, with the dawnward portion propagating ahead of the duskward one. Both segments expand duskward, reaching separate lengths of $18-25 d_{i}$, and both segments develop internal structures on east-west scales of $1-2 d_{j}$. The currents responsible for the ramp up of $B_{z}$ at the fronts are confined to narrow $\left(\lesssim d_{i}\right)$ ribbons whose localization is primarily associated with the electron $U_{\text {ey }}$ flow. The incoming ion flow is slowed down and deflected duskward at the front, and ambient ions are reflected back from the moving front. These processes create regions of enhanced $T_{i x x}$ both downstream and upstream of the front, while there is a local minimum at the front itself. These results help to explain the prevalence of $\sim 1 R_{E}$ flow jets in the plasma sheet.
\end{abstract}

Keywords: Localized magnetic reconnection; Dipolarization front; Reconnection jet breakup; Ballooning/interchange mode; 3D PIC simulation; Ion temperature asymmetries

\section{Findings Introduction}

It is well known that in the Earth's magnetotail, the bulk plasma flow speed is highly variable, with brief periods of fast plasma flows ("bursty bulk flows" (BBFs)) providing much of the sunward transport of mass, energy, and magnetic flux (Angelopoulos et al. 1992; Baumjohann et al. 1990). As a result of coordinated studies using multi-satellite observations (e.g., Angelopoulos et al. 1997; Nakamura 2004; Sergeev et al. 1996), it has been demonstrated that these fast flows are confined to flow channels in the plasma sheet whose full width is of the order of $1-3 R_{E}$ in the dawn-dusk direction and $1.5-2 R_{E}$ in the north-south direction.

A common feature of the BBFs observed in the nearEarth plasma sheet at $x \sim-10 R_{E}$ is the occurrence of transient dipolarizations at the leading edge of the flows (Ohtani et al. 2004). These structures exhibit a sharp increase of $B_{z}$ at the flow itself (giving rise to the name of "dipolarization front" (DF)) preceded frequently by a smaller negative $B_{z}$ variation. These dipolarizations are often interpreted as signatures of magnetic flux pileup

Correspondence: pritchet@physics.ucla.edu

Department of Physics and Astronomy, University of California, Los Angeles, 405 Hilgard Avenue, Los Angeles, California 90095-1547, USA
(Baumjohann et al. 1999; Hesse and Birn 1994; Shiokawa et al. 1997) or alternatively as resulting from a currentdriven instability (Lui et al. 1988). Observations with the radially separated THEMIS spacecraft enabled Runov et al. (2009) to identify the DFs as coherent plasma/flow structures that propagate predominantly earthward over a distance of at least $10 R_{E}$. The front thickness of the DF was found typically to be on the order of the ion inertial length (several hundred kilometers).

Numerous simulations of magnetic reconnection, usually in 2D, have shown that the outflow from an $\mathrm{X}$ line contains a leading edge region of magnetic field enhancement that is strikingly similar in many respects to the features of a DF. While there were some ambiguities in early simulations using periodic boundary conditions and/or rather small physical domain sizes as to whether this field enhancement was a real effect or an artifact of interactions with the boundaries, the use of larger simulation sizes and the development of open boundaries has shown that these structures are still present (e.g., Daughton et al. 2006; Pritchett 2010; Sitnov and Swisdak 2011). Thus, it is widely assumed that the DFs arise from magnetic reconnection.

Treatments of magnetic reconnection in 2D cannot of course address the issue of what physics leads to the

\section{Springer}


$1-3 R_{E}$ cross-tail extent of the BBFs. Recently, Pritchett (2015) employed 3D particle-in-cell (PIC) simulations in which magnetic reconnection was initiated in a localized cross-tail region of extent $4-24 d_{i}$, where $d_{i}$ is the ion inertia length based on the density in the current sheet. The chief finding was that initial reconnection regions with width $<12 d_{i}$ expanded in the ion-drift direction (duskward) to form a single front of width $15-20 d_{i}$ regardless of the initial width, thus explaining the $1 R_{E}$ minimum width of DFs. The resulting fronts were also observed to break up into small-scale finger structures of the order of $1-2 d_{i}$ in cross-tail extent. The $24 d_{i}$ width case, however, was found to separate into two segments, each in the $15-20 d_{i}$ range. In this paper, we explore in more detail this case of a large front breaking up into two sections, as opposed to the case of expanding small fronts emphasized in Pritchett (2015).

\section{Simulation method}

The present 3D PIC simulation employs the same methodology as described in Pritchett (2015). The simulation volume is $-64 \leq x / d_{i} \leq 64,0 \leq y / d_{i} \leq 64$, $-16 \leq z / d_{i} \leq 16$, where $d_{i}$ is the ion inertia length based on the peak density $n_{0}$ of the current-carrying Harris distribution. There is also a uniform background plasma with density $n_{b}=0.1 n_{0}$. The reconnection is initiated in a localized region $0 \leq x / d_{i} \leq 3.1,20 \leq y / d_{i} \leq 44$, $-2.1 \leq z / d_{i} \leq 2.1$ by blocking the cross-tail current of both the electrons and ions every 20 time steps (corresponding to an interval of $\sim 4 \omega_{p e}^{-1}$ or $0.03 \Omega_{i 0}^{-1}$, where $\omega_{p e}$ is the electron plasma frequency, $\Omega_{i 0}$ is the ion gyrofrequency in the $B_{0}$ field, and the mass ratio is $m_{i} / m_{e}=64$ ). The $x$ boundaries in the simulation are open such that all particles are removed and a thermal flux of new particles (one-sided Maxwellian) is injected continually back into the system. The $B_{z}$ field is allowed to propagate out of the system at speed $c$. At the $z$ boundaries, conducting conditions are assumed, and particles striking such a boundary are reintroduced in the opposite half $z$ plane with $v_{x}=-v_{x}$ and $v_{z}=v_{z}$. Periodicity in the $y$ direction is assumed for both the particles and fields. The coordinate system used in the simulations has $x$ increasing tailward, $y$ directed dawnward, and $z$ directed northward.

\section{Results}

\section{Overview of jet expansion}

The present simulation follows the self-consistent evolution of a reconnection front that is initiated with an initial cross-tail extent of $24 d_{i}$. For the initial interval of $\Omega_{i 0} t \lesssim 25$, the reconnection pulses as viewed in the equatorial plane propagate both earthward and tailward as relatively laminar structures, with only slight expansions at the duskward and dawnward edges and small enhancements in $\left|B_{z}\right|$ at the leading dawnward corners. Figure 1a

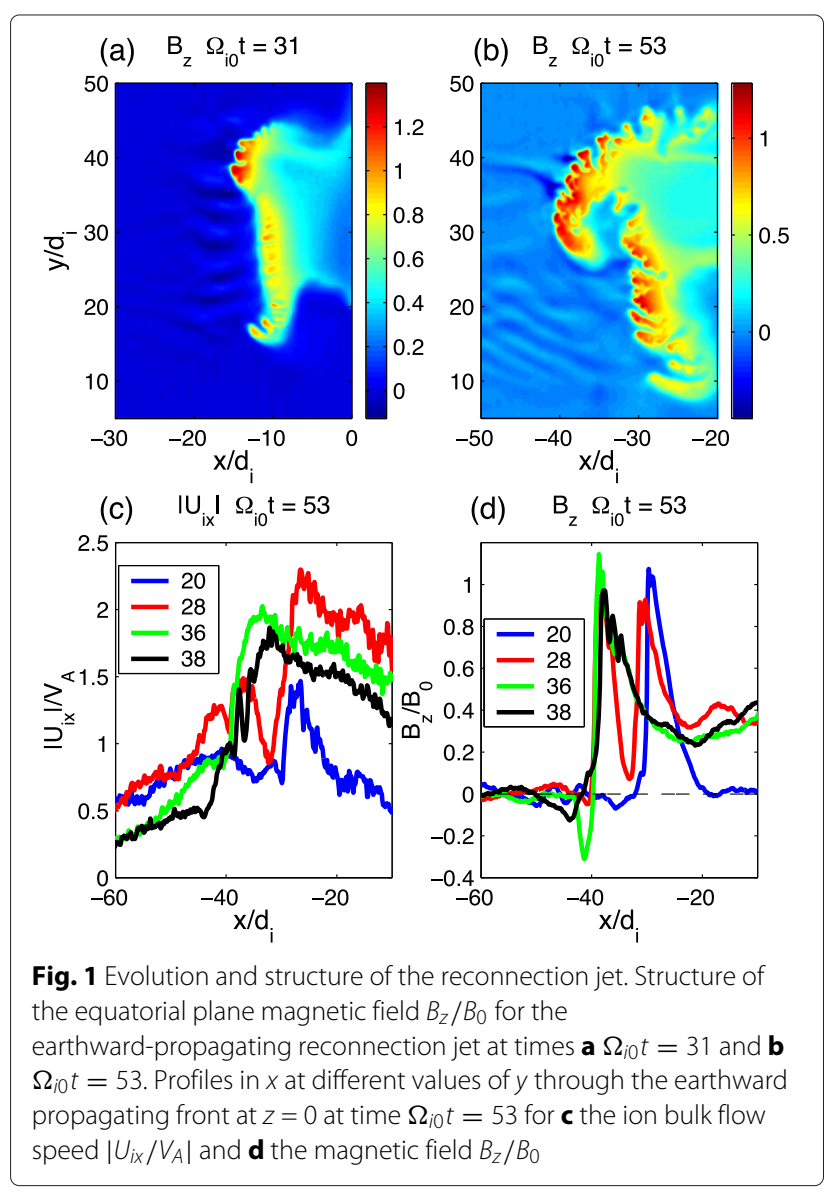

shows the earthward traveling $B_{z}$ pulse in the equatorial plane at the later time $\Omega_{i 0} t=31$. Several significant changes are now apparent. The front is starting to separate into two segments, with the portion in $35 \lesssim y / d_{i} \lesssim 43$ starting to move ahead of the rest of the front and with the strength of its entrained $B_{z}$ field increasing above that in the lagging segment. Also, a clear smaller-scale $\left(\sim 1-2 d_{i}\right)$ structure has appeared within both segments, and there is also a faint precursor modulation in $B_{z}$ on a similar scale visible ahead of the front. As discussed in Pritchett (2015), this smaller scale structure is consistent with the growth of the ballooning/interchange instability (BICI), but it is larger than the scale expected for the lower hybrid drift instability that peaks at wavelengths satisfying $k_{y} \rho_{e} \approx 1$ (e.g., Daughton 2003), which corresponds to a length of $0.3 d_{i}$.

Figure $1 \mathrm{~b}$ shows the $B_{z}$ structure of the front at the later time $\Omega_{i 0} t=53$. Now both segments have expanded in the duskward direction to reach a total cross-tail width of 15$20 d_{i}$ each, and the maximum $B_{z}$ values are comparable for each segment. The small-scale structuring has evolved to show a distinct dawnward-directed elongation. At still later times (not shown), the two main segments elongate 
further (up to $\sim 25 d_{i}$ ) and become more ragged but with no further increase in peak $B_{z}$.

Figure 1c, $\mathrm{d}$ shows profiles in $x$ at $z=0$ for four different values of $y$ at time $\Omega_{i 0} t=53$ for the bulk ion speed $\left|U_{i x}\right|$ and $B_{z}$, respectively. For all four $B_{z}$ profiles, there are negative precursor dips, ranging from small magnitudes of $\sim 0.03 B_{0}$ (including three repeated such dips for $\left.y / d_{i}=20\right)$ up to a large magnitude of $0.3 B_{0}$ for $y / d_{i}=$ 36 , as well as small positive blips. All of these features are typical of observed DFs, as demonstrated for example in the THEMIS P1 event of 27 February 2009 discussed by Runov et al. (2009). For the $B_{z}$ profile at $y / d_{i}=28$ (red curve), there is a second sharp increase tailward of the first (representing the lagging portion of the front) that is separated by a distance of $\sim 7 d_{i}$ from the first peak as well as a much weaker tertiary peak a further distance of $\sim 13 d_{i}$ behind the second. Such repeated positive fronts are also seen in the THEMIS P1 event of 27 February 2009, where the time interval between the first and second peaks is $\sim 13 \mathrm{~s}$. Using the observed earthward propagation speed of $330 \mathrm{~km} / \mathrm{s}$, this corresponds to a separation of $4300 \mathrm{~km}$ or about $10 d_{i}$ (using the density of $0.3 \mathrm{~cm}^{-3}$ ). This is quite consistent with the simulation result.

For the velocity profiles in Fig. 1c, the peak speeds of $\left|U_{i x}\right| / V_{A} \sim 1.5-2.2$ appear in back of the dawnward and duskward segments. These values are larger than the average speeds of $\sim 1 V_{A}$ of the two segments computed between the times shown in Fig. 1a, b. Thus the ion flow is being decelerated at the front itself (Drake et al. 2014;
Pritchett 2015). This increase in flow speed behind the main DF is also observed in the THEMIS P1 event, with a peak speed of nearly $1000 \mathrm{~km} / \mathrm{s}$ observed $45 \mathrm{~s}$ after the main DF. Note that P1 was located at $x=-20 R_{E}$, so that these results are for the mid-tail region rather than the near-Earth region near $-10 R_{E}$.

\section{Small-scale structure}

Figure 2 shows a blow up in the equatorial plane of the earthward traveling front at $\Omega_{i 0} t=53$ that illustrates the small-scale $\left(\sim 1-2 d_{i}\right)$ structures that develop. Figure $2 \mathrm{a}$ shows the $E_{x}$ field. Along the leading edge of the main front for $30 \lesssim y / d_{i} \lesssim 40$ are sharply localized regions of earthward directed field with magnitude $c E_{x} / v_{A} B_{0} \sim 1$ and $x$ extent $\sim 0.6 d_{i}$. A similar, although somewhat less pronounced, structure exists along the edge of the secondary front. Directly behind the main earthward $E_{x}$ field is a broader, patchier region of tailward directed $E_{x}$ of nearly comparable magnitude. There is thus a localized positive charge layer along the main front. Figure $2 \mathrm{~d}$ shows the $E_{y}$ field. There are strong enhancements in $E_{y}$ with width of $1-2 d_{i}$ in $y$ along both the main and secondary fronts. Thus, there is a net duskward $E_{y}$ field associated with the earthward drift of the fronts that is modulated by the small-scale BICI instability.

Figure 2b, e shows the current densities $J_{x}$ and $J_{y}$, respectively. The main ramp up of $B_{z}$ at both fronts is associated with a thin ( $\lesssim d_{i}$ scale) $J_{y}$ current layer. As is apparent from Fig. 2f, this localized layer is associated primarily

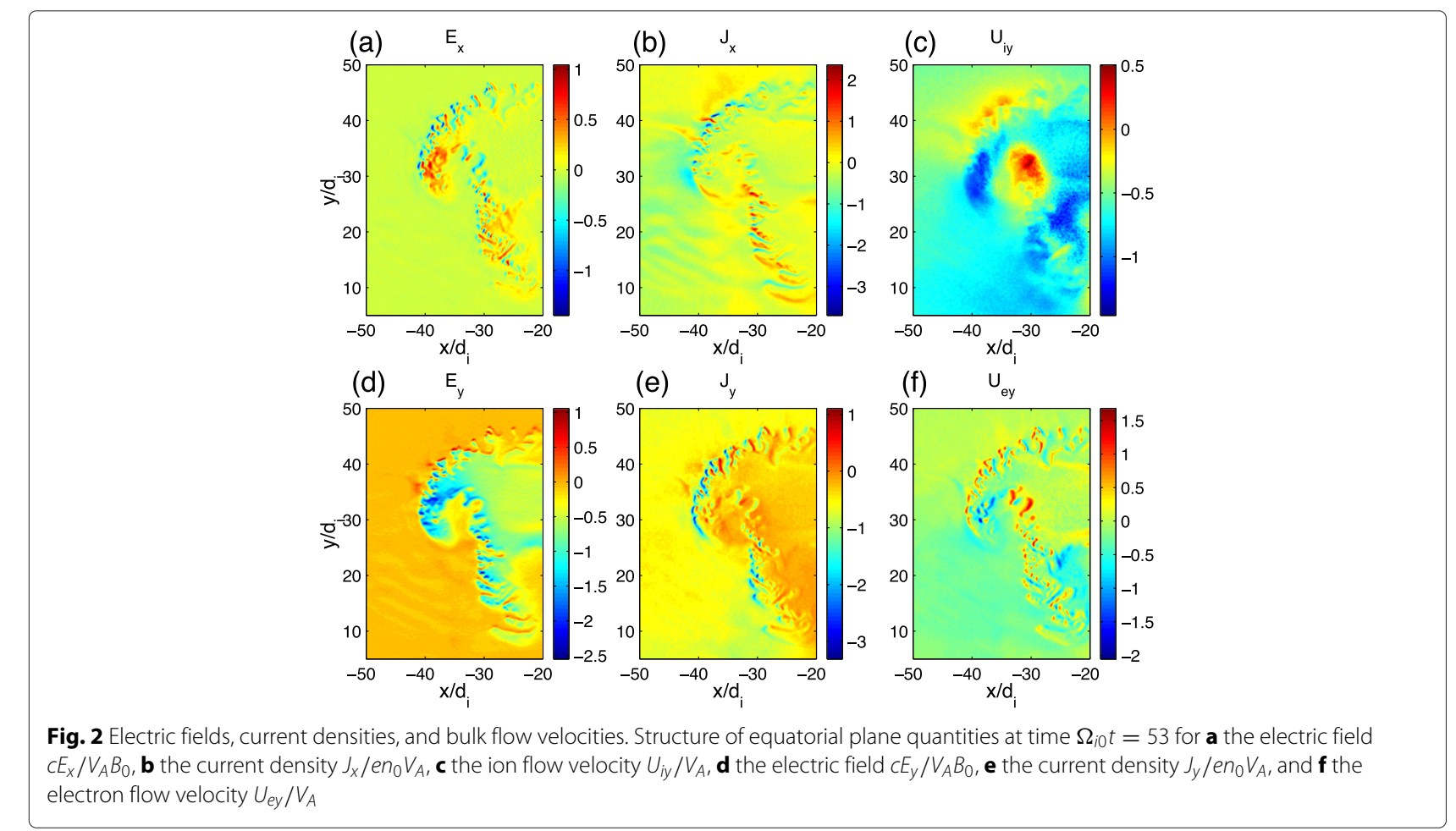


with narrow dawnward electron flows. The duskward ion flow is not negligible, however, amounting to about two thirds of the magnitude of the electron flow (Fig. 2c). In between the main and secondary fronts is a region $(27 \lesssim$ $\left.y / d_{i} \lesssim 33\right)$ of dawnward ion flow.

Figure 2b shows the $J_{x}$ current density. There are clear oscillations in $y$ ahead of both the main and secondary fronts associated with the electron flows driven by the BICI modes (Pritchett and Coroniti 2010). Together with the localized structures in $J_{y}$ ahead of the front, these $y$ dependent oscillations are responsible for the blips and dips in $B_{z}$ ahead of the fronts seen in Fig. 1d.

\section{Pressure and temperature}

Figure 3 shows plots in the equatorial plane at time $\Omega_{i 0} t=$ 53 for the ion pressure component $P_{i x x}$ (Fig. 3a), the density (Fig. 3b), the ion temperature $T_{i x x}$ (Fig. 3c), and the ion temperature $T_{i y y}$ (Fig. 3d). The density shows an enhancement by about a factor of 2 compared to the original equatorial plane density on the immediate downstream central and dawnward portions $\left(30 \lesssim y / d_{i} \lesssim 44\right)$ of the primary front. In contrast, the pressure enhancement is much larger (up to five to six times the original value) and is confined to the central portion $\left(30 \lesssim y / d_{i} \lesssim\right.$ 38 ) and extends much further downstream than does the

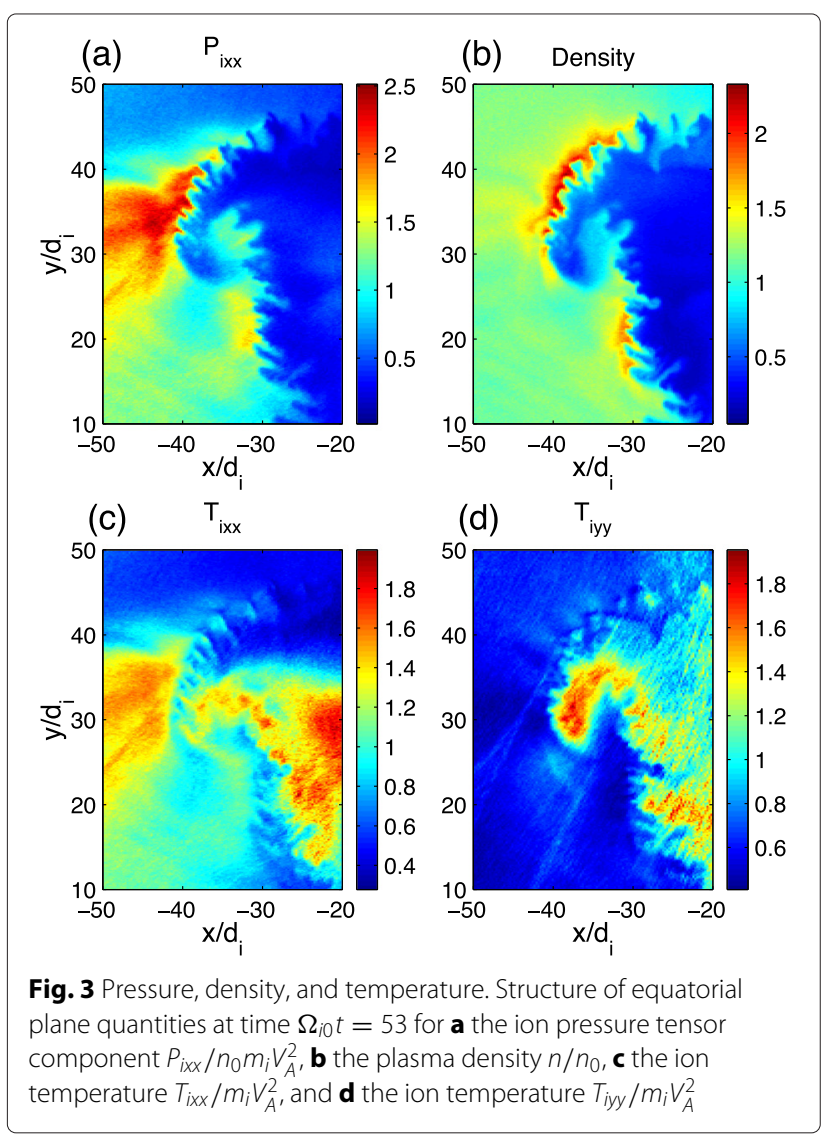

density enhancement. There are also considerably weaker enhancements in both $P_{i x x}$ and the density immediately ahead of the secondary front.

Some of these pressure and density features are consistent with the calculations of Zhou et al. 2014a, 2014b regarding the role of ion reflection in the asymmetric breaking and dawnward deflection of dipolarization fronts. However, as shown in Fig. 2c, the dominant ion $U_{i y}$ drift on the dawn side of the primary front is duskward with only two small regions of weak dawnward flow contained within the yellow region. Also, the $J_{y}$ current density in Fig. 2e does not have a single sign ahead of the main front. Rather, there are small regions of both signs that seem to be associated with the BICI structuring of the front. A dawnward directed ion current ahead of the main front would produce only a $B_{z}$ dip ahead of the front, whereas as shown in Fig. 1 there are positive blips as well as negative dips along the front. These seem more likely to be associated with the electron dynamics.

Figure $3 \mathrm{c}$ shows that the ion temperature $T_{i x x}$ is enhanced ahead of the main front on the center and dusk sides $\left(27 \lesssim y / d_{i} \lesssim 38\right)$ to about three to four times the initial value. There is likewise a weaker enhancement by a factor of about 2.5 ahead of the secondary front. Just upstream of both fronts is a region of reduced $T_{i x x}$, followed further upstream by an enhanced $T_{i x x}$. These temperature effects are non-gyrotropic since $T_{i y y}$ (Fig. 3d) shows no enhancement downstream of either front but does have a marked increase in the central region behind the main front. The heating for the electrons (not shown), however, is gyrotropic in the primary front with both $T_{\text {exx }}$ and $T_{\text {eyy }}$ increasing in the dawnward half by a factor of 5 relative to the initial value.

Figure 4 shows the ion $x, v_{x}$ phase space in the equatorial plane at three different values of $y / d_{i}=36,28$, and 20 at time $\Omega_{i 0} t=53$. The color scale is determined such that at each value of $x \int_{-\infty}^{\infty} f\left(v_{x}\right) d v_{x}=1$. Thus at a given value of $x$, each plot shows the corresponding ion velocity distribution function $f\left(v_{x} / v_{A}\right)$. In Fig. 4 a, which shows a cut through the dawnward front, there is an incident beam at $V_{i x} \sim-2 v_{A}$ that exists upstream of the front. At the front itself, the beam structure is reduced in speed and shows evidence of the beam breakup. On the earthward side of the front, there is a weak population of reflected and transmitted ions moving to the left with magnitude $\left|V_{i x}\right| \gtrsim 2 v_{A}$. This enhanced thermal spread is reflected in the enhanced $T_{i x x}$ temperature on the earthward side of the front (Fig. 3c). Figure $4 \mathrm{~b}$ shows a cut that passes through both the dawnward and duskward fronts. The region tailward of the duskward front $\left(x / d_{i}>-30\right)$ shows multiple beam structures, while the region between the two fronts $\left(-40<x / d_{i}<-30\right)$ shows the slowing down and breakup of the incident beams. Figure 4c, which passes only through the duskward front, nevertheless 


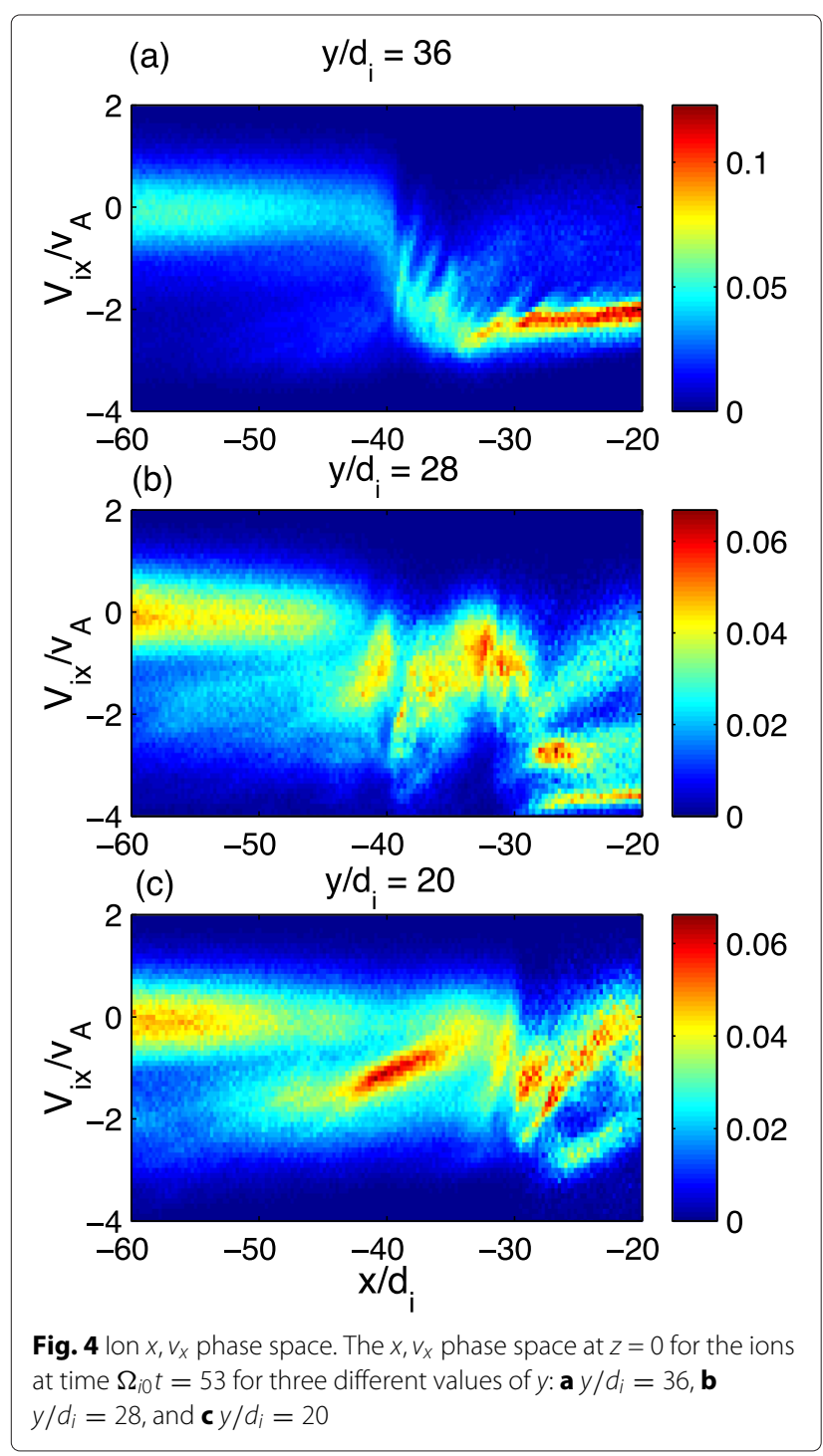

shows an extended structure at $x / d_{i} \approx-40$ and $V_{i x} / v_{A} \approx$ -1 that likely represents a duskward extension emanating from the dawnward front.

\section{Conclusions}

This paper has investigated the propagation of a reconnection jet in $3 \mathrm{D}$ produced from a source localized in all three dimensions. This was done by employing a 3D PIC simulation in which the cross-tail particle drifts in the source region were blocked periodically to represent the effective presence of a large anomalous resistivity that scatters the particles in the source region. The cross-tail width of the blocking region was $24 d_{i}$ or about $1.5 R_{E}$. The case of narrower blocking regions had been considered previously in Pritchett (2015).

In the initial stage $\left(\Omega_{i 0} t \lesssim 25\right)$, the reconnection fronts expanded both earthward and tailward as relatively laminar structures, a phenomenon that has been observed previously in numerous 2D simulations of reconnection. However, the fronts then started to separate into two segments, with the dawnward segment starting to move ahead and its entrained $B_{z}$ field strength increasing above that in the lagging segment. Both segments expanded duskward, reaching separate lengths of 18$25 d_{i}$. At the same time, both segments developed internal structures on east-west scales of $1-2 d_{i}$. This smaller scale structuring appears to be the result of the ballooning/interchange instability acting at the tailward $B_{z}$ gradient at the front of the jet (Pritchett and Coroniti 2010). Due to the expansion of the two jet segments and their differing speeds, it is possible that a slowly moving probe would encounter repeated dipolarization fronts with decreasing $B_{z}$ strength separated on scales of the order of $10-20 d_{i}$.

The currents responsible for the ramp up of $B_{z}$ at the jet fronts are confined to narrow $\left(\lesssim d_{i}\right)$ ribbons whose localization is primarily associated with the electron $U_{e y}$ flow. These currents exhibit structuring produced by the BICI mode, as do also the electric fields. Just downstream of the fronts are precursor structures in the current densities that are responsible for the commonly observed negative dips and positive blips in the $B_{z}$ time series plots.

There are pronounced increases in the density (by a factor of 2) and in the ion pressure component $P_{i x x}$ (by a factor of the order of 5) ahead of the primary (dawnward) front and weaker increases in both quantities ahead of the trailing (duskward) front. The density increase is associated with the slowing down of the beam front and the presence of reflected ambient ions at the front. The ion temperature $T_{i x x}$ downstream of the front is increased by a factor of the order of 4 and reflects the augmented thermal spread associated with the reflected and transmitted ions. Just behind each front is a local minimum in $T_{i x x}$, but this minimum value is still some $50-75 \%$ above the initial ambient value. The ion temperature $T_{i y y}$ is enhanced considerably behind the primary front on its duskward side. This is associated with an enhanced spread in $V_{i y}$ resulting from the enhanced cross-tail drift.

The present kinetic simulation of reconnectiongenerated fronts suggests that larger structures $>1 R_{E}$ in cross-tail extent will tend to break up to form two or more $\sim 1 R_{E}$ fronts. To fully confirm this effect will require completion of larger simulations with wider initial reconnection fronts. When combined with the previous results of Pritchett (2015) showing that fronts $<1 R_{E}$ tend to expand due to duskward propagation, it appears likely that $\sim 1 R_{E}$ should be a favored scale for reconnection fronts once they have been generated. This is consistent with the empirical evidence described in the Introduction. The mechanism(s) that is(are) responsible for the 
initiation of the reconnection events is still controversial, with both internal instabilities and external coupling processes being prime possibilities.

\section{Competing interests}

The author declares that he has no competing interests.

\section{Acknowledgements}

This research was supported by NASA grants NNX11AD74G and NNX14AF70G. The particle simulations in this work were made possible by allocations of advanced computing resources provided by the NASA High-End Computing (HEC) Program through the NASA Advanced Supercomputing (NAS) Division at Ames Research Center and by the National Science Foundation on Stampede at the Texas Advanced Computing Center.

Received: 18 March 2015 Accepted: 5 June 2015

Published: 27 June 2015

\section{References}

Angelopoulos V, Baumjohann W, Kennel CF, Coroniti FV, Kivelson MG, Pellat R, Walker RJ, Lühr H, Paschmann G (1992) Bursty bulk flows in the inner central plasma sheet. J Geophys Res 97:4027-4039

Angelopoulos V, et al (1997) Magnetotail flow bursts: association to global magnetospheric circulation, relationship to ionospheric activity and direct evidence for localization. Geophys Res Lett 24:2271-2274

Baumjohann W, Paschmann G, Lühr H (1990) Characteristics of high-speed ion flows in the plasma sheet. J Geophys Res 95:3801-3809

Baumjohann W, Hesse M, Kokubun S, Mukai T, Nagai T, Petrukovich AA (1999) Substorm dipolarization and recovery. J Geophys Res 104:24995-25000

Daughton W (2003) Electromagnetic properties of the lower hybrid drift instability in a thin current sheet. Phys Plasmas 10:3103-3119

Daughton W, Scudder J, Karimabadi H (2006) Fully kinetic simulations of undriven magnetic reconnection with open boundary conditions. Phys Plasmas 13(0272101)

Drake JF, Swisdak M, Cassak PA, Phan TD (2014) On the 3-d structure and dissipation of reconnection-driven flow bursts. Geophys Res Lett 41:3710-3716. doi:10.1002/2014GL060249

Hesse M, Birn J (1994) MHD modeling of magnetotail instability for localized resistivity. J Geophys Res 99:8565-8576

Lui ATY, Lopez RE, Krimigis SM, McEntire RW, Zanetti LJ, Potemra TA (1988) A case study of magnetospheric substorm models. Geophys Res Lett 15:721-4

Nakamura R, et al (2004) Spatial scale of high-speed flows in the plasma sheet observed by Cluster. Geophys Res Lett 31 (L09804) doi:10.1029/2004GL019558

Ohtani SI, Shay MA, Mukai T (2004) Temporal structure of the fast convective flow in the plasma sheet: comparison between observations and two-fluid simulations. J Geophys Res 109(A03210). doi:10.1029/2003JA010002

Pritchett PL (2010) Onset of magnetic reconnection in the presence of a normal magnetic field: realistic ion to electron mass ratio. J Geophys Res 115(A10208). doi:10.1029/2010JA015371

Pritchett, PL (2015) Structure of exhaust jets produced by magnetic reconnection localized in the out-of-plane direction. J Geophys Res 120:592-608. doi:10.1002/2014JA020795

Pritchett PL, Coroniti FV (2010) A kinetic ballooning/interchange instability in the magnetotail. J Geophys Res 115(A06301). doi:10.1029/2009JA014752

Runov A, Angelopoulos V, Sitnov MI, Sergeev VA, Bonnell J, McFadden JP, Larson D, Glassmeier KH, Auster U (2009) THEMIS observations of an earthward-propagating dipolarization front. Geophys Res Lett 36(L14106). doi:10.1029/2009GL038980

Sergeev VA, Angelopoulos V, Gosling JT, Cattell CA, Russell CT (1996) Detection of localized, plasma-depleted flux tubes or bubbles in the midtail plasma sheet. J Geophys Res 101:10817-10826

Shiokawa K, Baumjohann W, Haerendel G (1997) Braking of high speed flows in the near-Earth tail. Geophys Res Lett 24:1179-1182

Sitnov MI, Swisdak M (2011) Onset of collisionless magnetic reconnection in two-dimensional current sheets and the formation of dipolarization fronts. J Geophys Res 116(A12216). doi:10.1029/2011JA016920
Zhou XZ, Angelopoulos V, Liu J, Runov A, Li SS (2014a) On the origin of pressure and magnetic perturbations ahead of dipolarization fronts. J Geophys Res 119:211-220. doi:10.1002/2013JA019394

Zhou XZ, Angelopoulos V, Liu J, Runov A, Pan DX (2014b) Asymmetric braking and dawnward deflection of dipolarization fronts: effects of ion reflection. Geophys Res Lett 41:6996-7001. doi:10.1002/2014GL061794

\section{Submit your manuscript to a SpringerOpen ${ }^{\circ}$ journal and benefit from:}

- Convenient online submission

- Rigorous peer review

- Immediate publication on acceptance

- Open access: articles freely available online

- High visibility within the field

- Retaining the copyright to your article

Submit your next manuscript at $>$ springeropen.com 